\title{
Satisfacción de usuarios y profesionales en la evaluación de programas sociales*
}

\section{Customers' and professionals' satisfaction in social programs assessment*}

Núm. 7 (2016), pp. 116-140

Sánchez Pérez, Ma Carmen**

Recibido: abril, 2016

Aceptado: septiembre, 2016

JEL Clasif: I3 8

DOI: $10.5944 /$ reppp.7.2016.16352

\footnotetext{
Gracias a los servicios sociales municipales de los Ayuntamientos de Cuenca (AIS) y Albacete (SAS), por ofrecerme la posibilidad de participar en sus procesos de mejora y formar parte de los estudios que aquí se describen.

** Ma Carmen Sánchez Pérez. Universidad de Castilla-La Mancha. Facultad de Educación de Albacete. ORCID: http://orcid.org/oooo-ooo2-7195-7446.E-mail: mariacarmen.sanchez@uclm.es.
} 


\title{
Resumen
}

La satisfacción de los denominados clientes internos y externos por la mayoría de sistemas de gestión de calidad resulta ser un elemento de análisis imprescindible en cualquier proceso de evaluación y mejora de los servicios. Esta tendencia, arraigada en el ámbito privado, se ha extendido al espacio de los servicios públicos y, dentro de ellos, a la esfera de los servicios sociales.

En este artículo se plantea la importancia de desarrollar estudios de satisfacción en los servicios sociales básicos, tanto del conjunto de los profesionales, como de sus usuarios. Se observa una tendencia a ofrecer una valoración positiva sobre los servicios sociales básicos por las personas usuarias, como muestran diversos estudios demoscópicos sobre percepción de los servicios públicos. Del mismo modo, pese a ser escasos los estudios llevados a cabo con profesionales, destaca la elevada satisfacción expresada por quienes están en primera línea de los servicios sociales, lo que pone de manifiesto la vocación de servicio de estos trabajadores públicos.

Palabras clave: satisfacción; calidad; mejora; servicios sociales

\begin{abstract}
The study of customers' satisfaction is usually included in most of the quality management models and it is a relevant element in any process of assessment and improvement of services. This trend, deeply rooted in the private field, has also spread over the public services and in social services.

This paper sustains the relevance of developing satisfaction studies in general social services, not only from customers' perspective, but also from professionals. It is a proved trend that citizens make a good judgement of social services provision, as they show in several opinion polls about public services in Spain. On the same line, although there are little studies about people working in social services opinion, it is relevant the high satisfaction expressed by front line staff, that shows an important vocation in most of social services employees.
\end{abstract}

Key Words: satisfaction; quality; improvement; social services 


\section{Sobre el concepto de cliente interno $y$ externo: usuarios y profesionales}

Uno de los términos más utilizado al hablar de satisfacción en los diferentes modelos de gestión de calidad (EFQM, ISO, etc.) es el de "cliente», con una doble dimensión: externo (usuarios que reciben un servicio) e interno (profesionales que lo prestan) (AEVAL, 2009, p. 12).

Se considera fundamental para el éxito de una empresa conocer y orientar la organización hacia la satisfacción de las necesidades y expectativas, tanto actuales como futuras, de todas las partes interesadas: clientes y/o usuarios, personal y profesionales de la organización, dueños o inversores, proveedores y colaboradores, la sociedad en términos de comunidad y el público en general (AENOR, 2003, pp. 17-19). Superando el concepto de cliente, se habla de "partes interesadas» (por ejemplo, norma ISO 9004) cobrando una relevancia esencial para el desempeño de la organización los sistemas de medida de la satisfacción del cliente. De ahí que deba cuidarse el desarrollo y aplicación de herramientas para la recopilación de esa información, tanto de forma activa (encuestas, comunicación con usuarios, grupos de discusión, quejas), como pasiva (datos de prestación del servicio). Estos procesos, por los que las organizaciones solicitan, miden y siguen la retroalimentación de la satisfacción, deberían ofrecer información de forma continua para «escuchar la voz del cliente» de manera eficaz y eficiente (AENOR, 2003, p. 215 y ss.).

Del mismo modo, debe promoverse el conocimiento de las demandas y el reconocimiento de los profesionales de la organización, su satisfacción en el trabajo y su desarrollo personal, cuidando el ambiente laboral e intentando asegurar que el compromiso y la motivación sean lo más fuertes posibles (AENOR, 2003).

\subsection{Cliente interno: profesionales y personal del servicio.}

Desde el mundo de la empresa se dieron cuenta de que «los trabajadores son su bien más preciado y deben ser tratados como si fueran clientes para obtener el mayor rendimiento» (Prats Darder, 2005, p. 31). Peters and Waterman (1991), en su análisis de empresas con éxito, manifestaban la importancia de cuidar al cliente interno para poder garantizar la calidad al cliente externo. Albrecht (1998) resaltaba, así mismo, que para que las cosas funcionen fuera, primero deben ir bien dentro, otorgando un papel fundamental a la satisfacción de los trabajadores.

Estos consejos, válidos para el mundo de la empresa, forman también parte de los principios de la moderna gestión pública, en los que la visión del cliente interno es parte esencial de cualquier proceso de mejora, por lo que su satisfacción requiere de un estudio específico y una metodología propia (AEVAL, 2009, p. 12).

En cualquier modelo de calidad total orientado a la mejora debe considerarse la importancia de los clientes internos: profesionales que prestan los servicios y cuya satisfacción no sólo interesa ser conocida, sino que ha de ser cuidadosamente tomada en consideración en cualquier proceso de gestión. Quienes ostenten la dirección de los servicios deben tener en cuenta que la implicación y opinion de todos los grupos profesionales es 
imprescindible. Cuanto más motivados, satisfechos e implicados estén los profesionales con la institución, mejor atenderán a los usuarios que, a su vez, estarán más satisfechos.

\subsection{Clientes externos: personas usuarias y servicios sociales.}

En cuanto a los «clientes externos», cabe afirmar que la transformación del concepto de cliente al de usuario y, finalmente, ciudadano, está íntimamente ligada a la evolución de los sistemas de gestión de calidad.

En un primer momento, se utilizaba el término cliente (también consumidor), vinculándolo al ámbito empresarial-mercantil. Con la introducción de la calidad en el mundo de los servicios, se introduce una nueva concepción: la de usuario, ya que no siempre se producía el intercambio material de un bien, sino que la prestación del servicio consistía en el propio uso del mismo (Juanes \& Blanco, 2001). La siguiente transformación llega cuando la Administración Pública asume los planteamientos de calidad y mejora continua: ya no se trata de ofrecer calidad por un interés de mercado, sino como reconocimiento de un derecho que tienen los ciudadanos. Hemos de tener en cuenta la diversidad de perfiles atendidos por los servicios públicos que hace de la individualización en la atención, además de una exigencia técnica, una demanda explícita de los ciudadanos (Fernández Guerrero \& Martínez Moreno, 2003, p. 74).

Centrándonos en el sistema de servicios sociales, objeto de nuestra atención en este artículo, encontramos la convivencia de usuarios muy diversos: los que reclaman su derecho a acceder a un servicio; aquellos sobre los que la administración interviene «de oficio», para salvaguardar sus derechos; personas con una fuerte reticencia a reconocerse como usuarios de los servicios sociales (pues sigue afianzada en nuestra sociedad la idea de que los servicios sociales están dirigidos a grupos sociales «marginales»), etc.

En este sentido, Fernández Guerrero y Martínez Moreno (2003, p. 73) plantean que tener que recurrir a los servicios sociales todavía se experimenta como expresión de un fracaso, pues se sigue considerando que los recursos que habilita la Administración Pública están dirigidos a quienes no han podido resolver sus necesidades a través de la familia o el mercado. En palabras de un usuario:

«Yo pensaba que este tipo de servicios, pues bueno, como que está para otro tipo de gente, emigrantes o personas que realmente tuvieran más problemas que yo porque yo por lo menos tenía una familia, unos padres, una serie de circunstancias que bueno, que eran apropiadas, ¿̇no?» (Giménez Bertomeu et al., 2012, p. 66).

A muchas personas, por tanto, les resulta difícil identificarse como usuarias de estos servicios. Fruto de la propia evolución del sistema de servicios sociales, los primeros colectivos atendidos pertenecían mayoritariamente a grupos caracterizados por su extrema vulnerabilidad, con escasa capacidad de exigencia en cuanto a la calidad de los servicios ofrecidos. Sin embargo, la progresiva incorporación de las clases medias y la ampliación de los recursos sociales a necesidades más diversas ha transformado las demandas hacia los servicios, entendiéndose su prestación y mejora como un derecho ciudadano. 


\section{Elementos de análisis en el estudio de la satisfacción con los servicios}

Como se viene mencionando, el análisis de la satisfacción con los servicios recibidos aparece como uno de los elementos básicos en cualquier modelo de gestión (Juanes y Blanco, 2001, p. 94) y es considerado por muchos autores e instituciones (OMS, UNESCO, FEMP) como parte esencial de la calidad en las organizaciones (Vuori, Saturno Hernández, Delgado Vila, \& Suñol Sala, 1996). Este tipo de estudios se ha extendido tanto en el ámbito privado como, más recientemente, en los servicios públicos (MAP, 2006). Además de configurarse como un instrumento básico en la puesta en marcha de sistemas de gestión de calidad, es imprescindible que cuenten con un desarrollo continuado.

Tradicionalmente, se han considerado dos enfoques para la definición de la calidad del servicio desde las personas usuarias (MAP, 2006, p. 8 y ss.):

- Cumplimiento de «especificaciones» con las que el servicio fue diseñado.

- Cumplimiento de «expectativas» de los usuarios.

Tanto en una como en otra perspectiva, existen desajustes o «gaps» en el proceso de diseño y prestación del servicio, tal y como Zeithaml, Parasuraman y Berry (1993) plantean en su modelo. En su adaptación a la Administración Pública, el MAP (2006, p. 9) indica que pueden producirse tres tipos de desajustes, reflejados en la figura 1.

Figura I: Desajustes en la satisfacción de los usuarios.

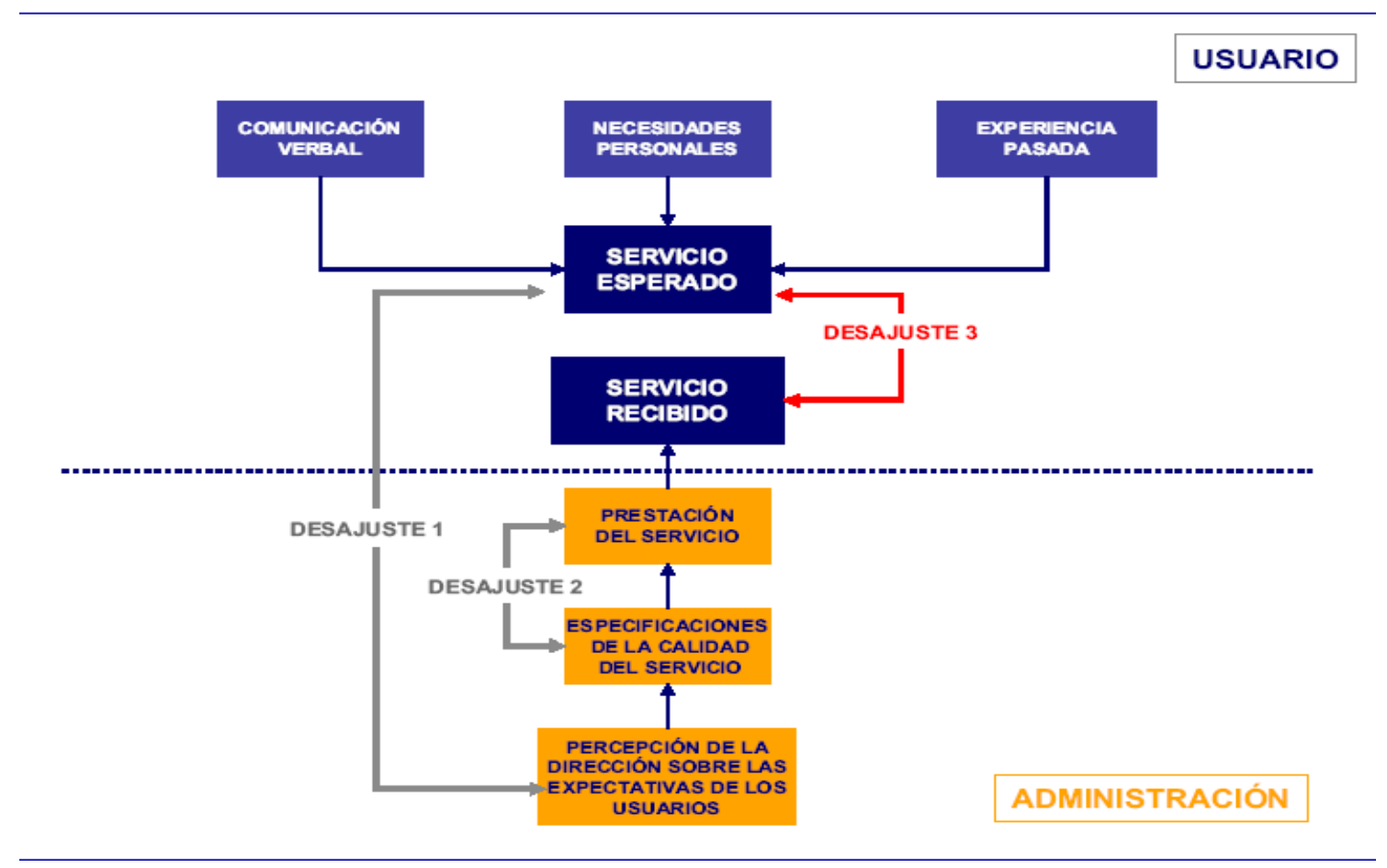

Fuente: Extraído de MAP (2006, p. 9). 
El desajuste 1 está provocado por las diferencias entre lo que los usuarios esperan del servicio y la percepción que la dirección tiene sobre esas expectativas. Suele producirse cuando la dirección da por hecho que conoce las necesidades de los usuarios y no escucha, ni a los usuarios ni a los empleados. En ocasiones, hay excesivos niveles entre la dirección y los empleados que prestan la atención, por lo que el flujo de información es difícil y llega distorsionado.

Cuando se produce una diferencia entre los planes del servicio y lo que realmente se ofrece, estamos ante el desajuste 2, provocado habitualmente por conflictos en la organización de quien presta el servicio (ambigüedad de las funciones, inadecuada supervisión y control, etc.).

El último desajuste (3) es consecuencia de los dos anteriores y el que genera más insatisfacción de los usuarios, ya que el servicio esperado no coincide con el recibido. Pone en relación expectativas con percepciones o resultados. Cabría establecer la siguiente fórmula:

$$
\frac{\text { Percepción }}{\text { Expectativa }}=\text { Satisfacción }
$$

Este denominado «paradigma de la desconfirmación» (MAP, 2006, p. 11) implica que la satisfacción del usuario depende directamente del nivel de resultado o prestación del servicio y está en relación inversa a la expectativa que tenga. Idénticos niveles de prestación del servicio producirán distintos grados de satisfacción y la acumulación de experiencias provocará cambios en el nivel de expectativas. Cabe hablar, por tanto, de varias dimensiones de la satisfacción: respecto a lo esperado, a lo recibido y a lo percibido.

Zeithaml et al. (1993) ofrecen un catálogo de variables en las que, con carácter general, se apoyan los usuarios para valorar la calidad de los servicios:

- Fiabilidad. Habilidad para ejecutar el servicio prometido de forma correcta y cuidadosa; hacer lo que se dice sin engaño, con puntualidad, etc.

- Competencia. Posesión por el profesional de las destrezas y el conocimiento requerido para la ejecución del servicio.

- Capacidad de respuesta. Disposición a atender a los clientes con un servicio rápido.

- Accesibilidad. Facilidad para localizar el servicio, comodidad para utilizarlo, horarios adecuados, dependencias fáciles de encontrar, contacto telefónico sencillo (líneas libres, derivación correcta, todas las llamadas atendidas), personas fácilmente localizables, servicios bien señalizados, tiempo de espera corto, posibilidad de realizar trámites por vía telemática, máxima desburocratización de la gestión, reducción de trámites.

- Empatía y cortesía. Atención, consideración, respeto y amabilidad del personal que atiende a los ciudadanos.

- Comunicación. Informar a los clientes con un lenguaje que puedan entender, escucharles.

- Credibilidad. Veracidad, creencia, honestidad en el servicio que se ofrece. 
- Seguridad. Inexistencia de peligros, riesgos o dudas; tranquilidad de sentirse en buenas manos. La seguridad no sólo es física; incluye también la confidencialidad de los datos, la confianza en el plano personal, etc.

- Comprensión hacia el ciudadano. Esfuerzo por conocer a los usuarios y sus necesidades; captar necesidades latentes, atender las quejas y sugerencias.

- Evidencia física del servicio. Apariencia de las instalaciones físicas, equipos, personal, materiales de comunicación. Son los elementos más tangibles del servicio.

Estas dimensiones quedarían sintetizadas en cinco: elementos tangibles, fiabilidad, capacidad de respuesta, seguridad y empatía, configurando uno de los modelos más extendidos y conocidos para medir la calidad: la escala SERVQUAL. Se obtiene una puntuación de cada uno de los criterios a través de un cuestionario, que incluye percepciones y expectativas. Su aplicación ofrece una calificación ponderada o no, que permite observaciones a lo largo del tiempo o entre entidades (Zeithaml et al., 1993, p. 205 y ss.).

Bengoa y Celorio (2003b, p. 141 y ss.) proponen considerar otros elementos, como aspectos éticos (honestidad del profesional, confiabilidad, sensibilidad, privacidad, veracidad, técnicos (competencia de los profesionales que atienden, atención conforme a estándares conocidos, seguridad, información comprensible, tangibilidad), elementos temporales (accesibilidad del servicio, tiempo y oportunidad del mismo, flexibilidad) y dimensiones psicológicas (reconocimiento personal por quienes atienden, amabilidad, estética ambiental).

No podemos obviar que la satisfacción percibida por el usuario es una valoración emocional y particular de carácter subjetivo, fruto de un proceso en el que compara su experiencia con otras previas, propias o de otros (Bengoa \& Celorio, 2003a, p. 142). Por ello, estos autores entienden que los principales criterios de calidad que valoran los usuarios, son: accesibilidad (atención suministrada del modo más rápido y cercano); efectividad (que consiga mejorar su situación); adecuación (en cuanto al estilo de comunicación, actitud del personal y ambiente en el que se ofrece el servicio) y receptividad a las quejas.

Salvador Pedraza (2000a, p. 243; 2003, p. 168) propone dos cuestiones más que cobran importancia en los servicios sociales: igualdad (percepción de que todo el mundo es tratado de la misma manera) y honradez (los ciudadanos han de percibir que los trabajadores del servicio no los utilizan en beneficio propio, con intereses partidistas, etc.).

Son clásicos los estudios realizados para conocer la satisfacción del cliente, sobre todo en el ámbito privado-empresarial, pero no se ha alcanzado un acuerdo sobre el constructo «satisfacción» (Cabrera Rodríguez, Donoso Vázquez, Aneas Álvarez, Campo Sorribas, \& Pi i Murugó, 2010; Cardozo, 1965). Se ha dejado de identificar el resultado final como único objeto de interés, para centrarse en aspectos relativos al proceso de prestación y su desarrollo, como plantean las propuestas de Parasuraman, Zeithmal y Berry (1994); Zeithaml, Parasuraman y Berry (1990), en línea con las tendencias de la nueva gestión pública (Alemán Bracho \& Pérez Sánchez, 2000; Barranco Expósito, 2004a; Pérez Menayo, 2005; Trinidad Requena, 2010; Viñas, 2005).

Pese a ser la satisfacción un complejo objeto de análisis, cabe resaltar los siguientes aspectos en su proceso de estudio: 
- Las propiedades del servicio, diseñadas desde un ámbito científico-técnico o político, orientadas a dar satisfacción a las necesidades de los usuarios. Son componentes que están presentes en todos los servicios personales y numerosos autores coinciden en identificar.

- Aspectos técnico-científicos, relativos a los conocimientos, juicios y destrezas del profesional en la intervención. Permitirán alcanzar un diagnóstico y propuesta de intervención, en base a los conocimientos científicos del profesional y su capacidad técnica.

- Relación interpersonal, que tiene máxima importancia, pues el intercambio de información, la comunicación profesional-usuario es el vehículo de la intervención social. Que se produzca con discreción, intimidad, tacto, empatía, respeto, etc. hace de esta interacción un elemento clave para la valoración de un buen servicio desde el punto de vista del usuario (Villegas Periñán \& Rosa Díaz, 2003, p. 51), complemento imprescindible a una buena calidad científico-técnica.

- Entorno, en palabras de Donabedian (1989, p. 103), «amenidades». Incluye la valoración de los elementos relativos a las infraestructuras e instalaciones del servicio, el confort de las mismas, los sistemas de información, etc.

- Las expectativas de los usuarios sobre cómo se prestará el servicio, con una base subjetiva y basada en opiniones recibidas por otros usuarios, en la comunicación del mercado, por experiencias previas en el servicio, etc.

- Y las percepciones del usuario sobre la atención recibida, basadas en la experiencia de su paso por el servicio, también con un fuerte componente subjetivo y afectivo.

Expectativas, percepciones y características del servicio configurarán elementos clave en el análisis de la satisfacción de usuarios, también en servicios sociales básicos (Martínez Tur, Ramos López, Marzo, \& Peiró Silla, 2002; Salvador Pedraza, 200ob; Sánchez, Martínez Tur, González Morales, Ramos López, \& Peiró Silla, 2009).

Han resultado bases teóricas esenciales para nuestro estudio de satisfacción de usuarios las concepciones de la calidad planteadas por Donabedian (1989) y Zeithmal et al. (1993). Así mismo, el modelo de la confirmación (o desconfirmación) de expectativas (Bolton \& Drew, 1991; Martínez Tur et al., 2002; Sánchez et al., 2009), permitirá detectar los desajustes o gaps, que apunten ámbitos de mejora para los servicios (Zamudio Igami, 2005; Zeithaml \& Parasuraman, 1994).

\section{El análisis de la satisfacción de usuarios y profesionales en el marco de procesos de mejora: estudios en los servicios sociales municipales}

Se describen en este artículo los resultados de sendos estudios llevados a cabo en el ámbito de los servicios sociales municipales; uno orientado a conocer la percepción y satisfacción de los usuarios de servicios sociales en Cuenca y, otro, la de los profesionales de primera línea de los servicios sociales municipales de Albacete. Se buscaba: 
- Identificar los elementos más relevantes en la construcción de la satisfacción de los usuarios y de los profesionales de servicios sociales, así como la capacidad explicativa de diferentes variables de análisis.

- Diseñar procedimientos e instrumentos de evaluación de la satisfacción de los ciudadanos usuarios y de los profesionales de los servicios sociales municipales, tomando en consideración, a modo de buenas prácticas, los elementos positivos de experiencias e investigaciones similares.

- Aplicar los procedimientos e instrumentos para obtener información relevante sobre el perfil y el nivel de satisfacción de la ciudadanía usuaria de los servicios sociales de Cuenca: conocer su opinión sobre los servicios, atención recibida, prestaciones sociales y programas dependientes de los servicios sociales municipales.

- Recopilar estudios de satisfacción de usuarios en servicios sociales básicos municipales, para comparar los resultados obtenidos.

- Aplicar los procedimientos e instrumentos para obtener información relevante sobre el perfil y el nivel de satisfacción laboral de los profesionales de primera línea de los servicios sociales de Albacete (grupos profesionales de conserjes-limpiadores, educadores y auxiliares administrativos).

- Analizar ${ }^{1}$ si existen diferencias significativas en la valoración de los servicios, en función de variables como sexo, edad, tipo de demanda (usuarios) o grupo profesional y antigüedad en el puesto de trabajo (profesionales).

- Extraer conclusiones para la mejora institucional y de los servicios, realizando propuestas fundamentadas de potenciales cambios a incorporar en la gestión de los servicios y prestaciones, que promuevan su mejora desde la perspectiva de los ciudadanos y profesionales.

Pasemos a conocer en detalle sus principales caracterísiticas y conclusiones.

\subsection{Satisfacción de usuarios como objeto de estudio en los servicios sociales básicos.}

Aunque los servicios sociales cuentan con elementos peculiares, los análisis que puedan realizarse sobre la satisfacción de sus usuarios serán similares a otros estudios de satisfacción con los servicios públicos y, más concretamente, con otros servicios del bienestar social, entre los cuales el uso del cuestionario es extendido. En los programas sociales, se ha venido a considerar a sus beneficiarios como «consumidores» y al servicio ofrecido como «producto» (Tornero, Ruiz, \& Miralles, 2011, p. 88), reconociéndose las dificultades en el análisis de la satisfacción (como en el resto de prestaciones de servicios, en las que se combinan elementos tangibles e intangibles). Sin embargo, cada vez más se ha puesto en valor la opinión de los usuarios, en su consideración de sujetos de derechos y con criterio para definir una opinión sobre la atención recibida (M. E. Medina Tornero, 1997; Pillinger \& Fundación Europea para la Mejora de las Condiciones de Vida y de Trabajo, 2001).

A pesar de ello, los estudios sobre la opinión de usuarios aún son escasos y relativamente recientes en el ámbito de atención de los servicios sociales básicos, en coherencia

$1 \quad$ La mecanización y análisis de datos cuantitativos de ambos cuestionarios se realizó con el programa IBM SPSS Statistics 19. Para la explotación de la información cualitativa (entrevistas a informantes clave, grupos de discusión), se ha utilizado el programa ATLAS.ti 6.

Revista de Evaluación de Programas y Políticas Públicas | Núm. 7 (2016), pp. 116-140 
con el escaso valor otorgado a la evaluación de los servicios de la Administración Pública, entendida en un sentido amplio, y con las resistencias que genera, como plantean Alemán Bracho y Trinidad Requena (2012, pp. 55, 84-86); Trinidad Requena y García Moreno (2012). Ello no implica que la administración no realice evaluaciones de sus programas y servicios, sino que en múltiples ocasiones, los datos obtenidos no llegan a sistematizarse en los correspondientes informes de resultados, ni mucho menos ven la luz mediante su difusión desde una perspectiva académica (Pérez Cosín \& Deslauriers, 2004).

La satisfacción de personas usuarias ha ido logrando un progresivo avance, inicialmente en los servicios sociales especializados (Barranco Expósito, 2000; García Sánchez, Mirete Ruiz, Marín Oller, \& Romero, 2008; Imserso, 1998, 1999; Literas Rondón, Navarro, \& Fontanals de Nadal, 2010) y más recientemente en los básicos. En este sentido, ha sido el servicio de ayuda a domicilio la prestación básica que más visibilidad ha tenido en la difusión de los resultados sobre la opinión de sus usuarios acerca de los servicios (Medina Tonero, 2000, 2003; Robles Ríos \& Lara Martínez, 2009).

Se consideró de especial interés para este trabajo analizar el servicio de Información, Valoración y Orientación ya que, dentro de los servicios sociales básicos, es una prestación que goza de una intensa relación profesional. Se trata de un servicio que, atendido por profesionales del Trabajo Social, recibe demandas de atención cada vez más diversas y complejas, que requieren de una intervención integral (Aguilar Hendrickson, 2013; Casado Pérez, 2007; De La Red Vega \& Conde Rodríguez, 2000; Fantova Azcoaga, 2008).

Se presenta en la siguiente tabla una síntesis de los estudios identificados en este ámbito, cuyos resultados han sido tomados en consideración para la discusión de los nuestros. 
Tabla I. Estudios sobre satisfacción de usuarios de los servicios sociales básicos desarrollados en España.

\begin{tabular}{|c|c|c|}
\hline AUTOR & ÁMBITO ESTUDIO & $\begin{array}{l}\text { CARACTERISTICAS DEL } \\
\text { ESTUDIO }\end{array}$ \\
\hline $\begin{array}{l}\text { Servicio de Acción Social del } \\
\text { Ayuntamiento de Albacete } \\
(2016)\end{array}$ & $\begin{array}{l}\text { Usuarios del Servicio de Información, } \\
\text { Valoración y Orientación. }\end{array}$ & $\begin{array}{l}\text { Adaptación SERVQUAL ( } 24 \text { items). } \\
\text { Escala I-I0. } \\
\text { Muestra } 57 \text { I personas. Entrevista a la } \\
\text { salida de la atención. }\end{array}$ \\
\hline Arenas Martínez (2015) & $\begin{array}{l}\text { Calidad percibida por los usuarios. } \\
\text { Cuatro centros de servicios sociales } \\
\text { de zona de Avilés (Asturias, España) }\end{array}$ & $\begin{array}{l}\text { Adaptación de cuestionario SERVQUAL. } \\
\text { Escala I-I0. } \\
\text { Entrevista representativa de } 104 \text { per- } \\
\text { sonas que han accedido a los servicios } \\
\text { en los últimos } 4 \text { años. }\end{array}$ \\
\hline Peña Salas (20|4) & $\begin{array}{l}\text { Personas atendidas en los servicios } \\
\text { sociales comunitarios del Centro } \\
\text { Municipal de Servicios Sociales Delicias } \\
\text { del Ayuntamiento de Zaragoza. }\end{array}$ & $\begin{array}{l}\text { Muestra } 212 \text { casos. } \\
\text { Cuestionario SERVQUAL. } \\
\text { Escala I-5. }\end{array}$ \\
\hline $\begin{array}{l}\text { Giménez Bertomeu, Lillo } \\
\text { Beneyto, Doménech López y } \\
\text { Lorenzo García (2012) }\end{array}$ & $\begin{array}{l}\text { Indicadores de Calidad en los Servicios } \\
\text { Sociales, con usuarios de tres Centros } \\
\text { Municipales de Servicios Sociales } \\
\text { Comunitarios de Alicante. }\end{array}$ & $\begin{array}{l}6 \mathrm{I} \text { usuarios. } \\
\text { Entrevistas en profundidad. } \\
\text { Definición de estándares de calidad. }\end{array}$ \\
\hline $\begin{array}{l}\text { Medina Tornero and Medina } \\
\text { Ruiz (20II) }\end{array}$ & $\begin{array}{l}\text { Centros de servicios sociales del } \\
\text { Ayuntamiento de Murcia ( } 2 \text { centros). }\end{array}$ & $\begin{array}{l}\text { Estudio sobre el análisis de la calidad } \\
\text { percibida en usuarios de servicios } \\
\text { sociales Comunitarios; índice general } \\
\text { de satisfacción utilizando la escala } \\
\text { SERVQUAL. }\end{array}$ \\
\hline Sánchez Aguado (201I) & $\begin{array}{l}\text { Ayuntamiento de Valdemoro (Madrid), } \\
\text { a través del INTRESS }\end{array}$ & $\begin{array}{l}\text { Creación de mapa de procesos que } \\
\text { incluía medición de satisfacción de } \\
\text { los usuarios de servicios sociales (por } \\
\text { desarrollar). }\end{array}$ \\
\hline $\begin{array}{l}\text { Ayuntamiento de Madrid } \\
(2010)\end{array}$ & $\begin{array}{l}\text { Estudio de satisfacción en Servicios } \\
\text { Sociales Comunitarios. }\end{array}$ & $\begin{array}{l}\text { Cuestionario estructurado. } 15 \text { preguntas. } \\
\text { Entrevista a la salida de los centros } \\
\text { sociales. } \\
\text { I } 577 \text { usuarios de centros de servicios } \\
\text { sociales. } \\
2 \text { I distritos. }\end{array}$ \\
\hline $\begin{array}{l}\text { García Pineda y Gago } \\
\text { Cabrera (2010) }\end{array}$ & $\begin{array}{l}\text { Centro de servicios sociales. Servicio } \\
\text { Social de base del Ayuntamiento de } \\
\text { San Pedro del Pinatar (Murcia). }\end{array}$ & $\begin{array}{l}\text { Estudio sobre el perfil de usuarios y su } \\
\text { satisfacción. }\end{array}$ \\
\hline $\begin{array}{l}\text { Siis. Dokumentazio eta } \\
\text { lkerketa Zentroa Centro de } \\
\text { Documentación y Estudios } \\
(2010)\end{array}$ & Comunidad Autónoma del País Vasco. & $\begin{array}{l}\text { Estudio sobre la implantación de siste- } \\
\text { mas de calidad en los servicios locales. } \\
\text { Encuestas a las y los usuarios de servi- } \\
\text { cios sociales. }\end{array}$ \\
\hline FOESSA (2009) & Hogares españoles. & $\begin{array}{l}\text { Encuesta FOESSA } 2008 \text {. Preguntas } 107 \\
\text { y } 108 \text {. Uso y satisfacción con los servi- } \\
\text { cios sociales. } 1246 \text { personas. } \\
\text { Escala satisfacción (respuesta } 4 \text { opcio- } \\
\text { nes). Presentación o no de diversos } \\
\text { problemas en la prestación de servicio. }\end{array}$ \\
\hline $\begin{array}{l}\text { Del Pozo Herráiz y Sánchez } \\
\text { Pérez (2010) }\end{array}$ & Usuarios SIVO Cuenca & $\begin{array}{l}\text { Encuesta propia. } 29 \text { I respuestas. } \\
6 \text { Oleadas (2007-2010). } \\
\text { Escala I a 5, preguntas de respuesta } \\
\text { cerrada y abierta. }\end{array}$ \\
\hline Moya Vaquero (2000) & $\begin{array}{l}\text { Programa de Mejora continua de la } \\
\text { calidad en el Centro Municipal de } \\
\text { Servicios Sociales Comunitarios de } \\
\text { Alcázar de San Juan (Ciudad Real). }\end{array}$ & $\begin{array}{l}\text { Encuesta telefónica. } \\
150-170 \text { usuarios. }\end{array}$ \\
\hline
\end{tabular}

Fuente: elaboración propia a partir de las referencias indicadas. 


\subsection{Satisfacción de usuarios del Servicio de Información, Valoración y Orientación (SIVO) en Cuenca.}

En el estudio de los servicios sociales municipales de Cuenca, se seleccionó el SIVO por ser un servicio que recibe demandas de todo tipo y atiende a usuarios de muy diversas características en un gran volumen de atención. Dado su carácter de «puerta de entrada» al conjunto de los servicios sociales, la valoración que del mismo realicen sus usuarios resultará de gran trascendencia para el conjunto del sistema.

La metodología seleccionada fue el cuestionario, medio habitual para conocer la valoración que hacen los usuarios sobre los servicios recibidos (MAP, 2006; Vavra, 2002), como puede comprobarse en la tabla 1. Tras el análisis de estudios similares, se optó por diseñar un modelo de cuestionario propio, con las siguientes dimensiones de análisis:

Tabla 2. Dimensiones de análisis en el cuestionario de satisfacción elaborado.

\begin{tabular}{|c|c|}
\hline DIMENSION & CUESTIONES \\
\hline \multirow{5}{*}{ Datos personales y de aplicación del cuestionario. } & Edad. \\
\hline & Sexo. \\
\hline & Perfil (inmigrante o no). \\
\hline & Tipo de atención recibida (primera atención/en zona). \\
\hline & Lugar de aplicación cuestionario. \\
\hline \multirow{4}{*}{ Conocimiento y acceso a los servicios sociales. } & Conocimiento nombre profesional. \\
\hline & Valoración cambio profesional (si procede). \\
\hline & Conocimiento previo servicios sociales. \\
\hline & Modo de acceso a los servicios sociales. \\
\hline \multirow{3}{*}{ Proceso solicitud de cita. } & Modalidad de solicitud de cita. \\
\hline & Valoración sistema y personal de cita previa. \\
\hline & Tiempo de espera para la atención y valoración. \\
\hline \multirow{2}{*}{ Instalaciones. } & Acceso al centro. \\
\hline & Valoración instalaciones (comodidad, limpieza, intimidad). \\
\hline \multirow{4}{*}{ Atención trabajadora social. } & Horario de atención y su cumplimiento. \\
\hline & Desarrollo y duración de la atención. \\
\hline & Solicitud de documentación. \\
\hline & Satisfacción con la atención recibida. \\
\hline \multirow{4}{*}{ Satisfacción de expectativas. } & Motivo de consulta. \\
\hline & Actuación ofrecida. \\
\hline & Valoración atención de la demanda. \\
\hline & Conocimiento sistemas de quejas y sugerencias. \\
\hline \multirow{3}{*}{ Satisfacción general con el servicio. } & Lo mejor. \\
\hline & Lo peor. \\
\hline & Sugerencias. \\
\hline
\end{tabular}

Fuente: elaboración propia con datos del estudio. 
Según la cuestión planteada, se seleccionaron distintos tipos de respuesta: cerrada, de opción múltiple, abiertas -para expresar opiniones o justificar respuestas- y de escala tipo Likert (de 1 a 5 , identificando las valoraciones 4 y 5 como bastante/muy satisfactorio y los valores 1 y 2 como nada/poco satisfactorio).

Este cuestionario se pilotó inicialmente con un pretest a la salida de la atención de la Trabajadora Social, para mejorar su comprensión y presentación. También se sometió a la consideración de expertos, técnicos de los servicios sociales y del mundo académico. Posteriormente, se procedió a su aplicación en los centros sociales (marzo 2007- noviembre 2010), mediante entrevista personal a los usuarios que habían recibido la atención de la Trabajadora Social, entendiendo que las personas «siempre estarán más dispuestas a contestar a una persona que a un frío papel» (Prats Darder, 2005). La siguiente tabla muestra el nivel de respuesta alcanzado:

Tabla 3. Grado de respuesta al cuestionario.

\begin{tabular}{|l|c|c|c|}
\hline & $\begin{array}{c}\text { USUARIOS } \\
\text { CONSULTADOS }\end{array}$ & RESPONDEN & \% Respuesta \\
\hline Oleada I & 312 & 51 & 16 \\
\hline Oleada 2 & 102 & 70 & 68,6 \\
\hline Oleada 3 & 80 & 56 & 70 \\
\hline Oleada 4 & 87 & 52 & 59,8 \\
\hline Oleada 5 & 79 & 47 & 59,5 \\
\hline Oleada 6 & 32 & 15 & 46,9 \\
\hline
\end{tabular}

Fuente: elaboración propia con datos del estudio.

Destacar de los datos obtenidos el elevado grado de satisfacción general con la atención recibida por la Trabajadora Social, pues un 91,7 \% de los usuarios manifestaba estar bastante o muy satisfecho. La valoración media del conjunto de datos recabados (oleadas 1 a 6) es de 4,54 sobre 5 (con una desviación de 0,822 ). La atención directa de la profesional del Trabajo Social sería un elemento clave en este servicio

Destaca la intensa relación de tres variables en la atención profesional: el trato agradable e individual, su adecuación a la demanda, junto con la sencillez en la información transmitida (valores de correlación de Pearson próximos al o,8 y significatividad a nivel o,o1 bilateral).

En cuanto a la satisfacción en relación con sus expectativas, encontramos que el servicio recibido ha sido valorado como mejor o mucho mejor de lo esperado por un $38 \%$ de los usuarios, siendo mayoritario el grupo de quienes la valoraron como más o menos igual a lo esperado (49,3\%). Podemos afirmar que se cumple en nuestra muestra el ya citado paradigma de la desconfirmación, en el que la satisfacción se ve condicionada tanto por las expectativas previas (bajas, en este caso) y la percepción sobre el servicio recibido (buena valoración).

Un 73,8 \% de los usuarios se manifestaron como «bastante o muy satisfechos/as» con la respuesta ofrecida por el servicio; la valoración media para esta cuestión fue de 3,9 sobre 5 (desv. típica de 1,16). 
Uno de los aspectos peor valorados en el conjunto del cuestionario fue la variable relativa al tiempo que pasó desde la presentación de la solicitud hasta que se obtuvo la respuesta, considerado como «nada o poco adecuado» por un 42,6 \% de los usuarios, con una puntuación media de 2,76 sobre 5 (desv. típica de 1,37). También aparecieron como elemenos menos valorados del servicio la burocracia o papeleos que implicaba en muchas ocasiones, así como el tiempo de espera para la cita, consistentes con otros estudios similares. En ese sentido se ofrecían propuestas de mejora: reducir tiempos de espera, mejorar las instalaciones, incremento de las prestaciones.

Se realizó un análisis de correlación, buscando relaciones estadísticamente significativas entre los resultados obtenidos y variables como edad, género y nacionalidad. No se han obtenido datos relevantes que permitan afirmar que existen diferencias significativas en las valoraciones que los distintos grupos de población realizan sobre el servicio, encontrándose únicamente leves variaciones puntuales, que no pueden calificarse de significativas. Este hecho es consistente con los resultados obtenidos en estudios similares, que tampoco ofrecen diferencias significativas debidas a variables sociodemográficas, como se comentará a continuación.

\section{3•3. Discusión de resultados.}

Los resultados de nuestro estudio coinciden con estudios similares, recogidos en la tabla 1. Independientemente de la escala utilizada, todos los estudios coinciden en ofrecer unas elevadas valoraciones sobre los servicios recibidos y, dentro de los elementos mejor considerados, la relación con la profesional. El trato amable y próximo, las habilidades y cualificación profesional y su implicación, son variables destacadas de modo reiterado. Si bien los espacios físicos aparecen en ocasiones como un aspecto importante (García Pineda \& Gago Cabrera, 2010), la tendencia muestra una mayor importancia concedida a la atención profesional que a las instalaciones. No obstante, aunque se consideren adecuadas, se apuntan propuestas orientadas a una mejora en la imagen, la accesibilidad y una mayor garantía de la confidencialidad en el servicio.

Otras cuestiones que, pese a la alta valoración del conjunto, apuntan posibles ámbitos de mejora son: la tardanza en la atención (tiempo de espera, habitualmente por una saturación en la demanda del servicio), la capacidad de respuesta (aunque los usuarios se sienten satisfechos con la atención recibida, opinan que la resolución a su demanda tarda en llegar) y un horario más flexible, que se ajuste a las posibilidades de los usuarios (llama la atención que en el estudio del Ayuntamiento de Madrid (2010), la satisfacción era aún mayor en el turno de tarde). La continuidad en los servicios y de los profesionales también son demandados por las y los usuarios (Giménez Bertomeu et al., 2012); se valora que el servicio se preste sin demora y los trámites se simplifiquen.

La seguridad, basada en profesionales cualificados; la confianza, fundada en la claridad de la información que se ofrece y la empatía, conseguida por un trato personalizado, son elementos que se reiteran como claves en el servicio.

Resulta llamativo que, al igual que ha ocurrido en nuestro estudio, apenas se encuentran diferencias significativas en las valoraciones en un análisis por género, estado civil, procedencia, situación laboral o edad (aunque en algunos estudios esta variable mostraba matices en aspectos concretos). 


\subsection{Satisfacción profesional y calidad en la Administración Pública. Los profesionales de primera línea en Albacete.}

En cuanto al estudio de la satisfacción de los clientes internos, de los profesionales, cabe afirmar que el análisis de la satisfacción profesional es un tema clásico en el ámbito de las organizaciones (Castillo Alonso \& Prieto Rodríguez, 1983; Fuentes García, Sánchez Cañizares, \& Artacho Ruiz, 2008; Lucas Marín, 1981). Sin embargo, la perspectiva propuesta en este trabajo aporta un elemento adicional: se sustenta en una concepción de los servicios públicos que, como venimos comentando, considera la evaluación de la satisfacción una obligación de las administraciones para promover la gestión de calidad, no sólo a nivel conceptual, sino también jurídico² ${ }^{2}$.

En este campo de estudio, cabría centrar la atención en la satisfacción que generan variables intrínsecas a la organización y relativas a su funcionamiento (Herzberg, 2010) como los elementos personales o de las condiciones de trabajo. Nuestro estudio, al enmarcarse en un proceso de calidad y mejora institucional, puso su foco de atención en las variables de la organización, en la línea de las teorías del enriquecimiento del trabajo individual (Hackman \& Oldham, 1976) y del puesto de trabajo de Bowen y Lawer, 1992 (Sánchez Cañizares, 2006, p. 130).

En el ámbito de los servicios sociales, aunque aún escasos, son de creciente interés los análisis para identificar y conocer la satisfacción de los y las trabajadoras, considerados por los diversos modelos de gestión de calidad «clientes internos» (AEVAL, 2014; Giménez Bertomeu \& Doménech López, 2012). Los estudios de satisfacción profesional realizados en España se han orientado principalmente a trabajadores sociales (Gómez García, 2013; Martín López, Padial Díaz, Romero Gallardo, \& Serrano Guerrero, 1996), centrándose, en muchos casos, en el fenómeno del burnout (Barranco Expósito, 2004b; Barrera Algarín, Malagón Bernal, \& Sarasola Sánchez-Serrano, 2015; Cosano Rivas, Hombrados Mendieta, \& Castilla Mora, 2014; Domínguez González \& Jaureguibehere, 2012; Esteban Ramiro, 2014; Facal Fondo, 2012; Fuente Roldán \& Sánchez Moreno, 2012).

Partiendo de esta realidad, en el Servicio de Acción Social del Ayuntamiento de Albacete (SAS) se desarrolló un estudio que tomaba en consideración a un grupo profesional bastante «invisible» en los procesos de mejora y en el día a día de los servicios. Buscaba conocer la satisfacción laboral de un grupo profesional al que apenas se ha prestado atención: trabajadores con los perfiles profesionales de conserjes de los centros de servicios sociales, auxiliares administrativas y educadoras-cuidadoras de centros especializados municipales ${ }^{3}$, primera línea de atención de las personas usuarias, en la «epidermis» de la administración. Estos colectivos, protagonistas de nuestro análisis, están presentes en procesos de apoyo a los servicios sociales y desarrollan una intervención de enorme transcendencia, tanto para el eficaz desarrollo del servicio como para la percepción que la población usuaria tenga del mismo. Son en muchas ocasiones las primeras personas que acogen y atienden a la ciudadanía que se acerca a los servicios sociales con una demanda de atención.

2 Real Decreto 951/2005, de 29 de julio, por el que se establece el marco general para la mejora de la calidad en la Administración General del Estado. BOE núm. 211 de 03 de Septiembre de 2005.

3 Colectivos que en el ámbito del SAS pertenecen a agrupaciones profesionales y grupo de clasificación C2. 
Desde la Comisión Europea se resalta la particular relevancia de conocer las percepciones de los y las profesionales de primera atención (que denomina front-line staff), pues su contacto directo con las necesidades de la ciudadanía les sitúa en una posición privilegiada, convirtiéndoles en colectivos de atención preferente en cualquier análisis de satisfacción laboral (Pillinger \& Fundación Europea para la Mejora de las Condiciones de Vida y de Trabajo, 2001, p. 14).

Como opción metodológica, se decidió aplicar un cuestionario individual auto-cumplimentado (Castillo Alonso \& Prieto Rodríguez, 1981), complementado con grupos de discusión, celebrados a continuación. También en este caso, a partir de la consulta y revisión de modelos existentes se diseñó un instrumento propio, adaptado a las peculiaridades del servicio e integrado en el proceso de mejora que se estaba desarrollando (Sánchez Pérez \& Aguilar, 2016), buscando identificar áreas críticas para promover la calidad del servicio. El cuestionario se estructuró en veinticinco preguntas, combinando respuestas abiertas, cerradas y de escala.

Tabla 4. Variables analizadas en el cuestionario del Servicio de Acción Social de Albacete.

\begin{tabular}{|c|}
\hline VARIABLES SOCIODEMOGRÁFICASY PROFESIONALES \\
\hline Sexo. \\
\hline Edad. \\
\hline Nivel de estudios. \\
\hline Antigüedad laboral. \\
\hline
\end{tabular}

\begin{tabular}{|c|l|}
\hline \multicolumn{2}{|c|}{ SATISFACCIÓN ASPECTOS ORGANIZATIVOS SAS. } \\
\hline & Organización interna del centro. \\
Organización interna del SAS. \\
Organización del trabajo. & $\begin{array}{l}\text { Adecuación del servicio y espacios. } \\
\text { Centro de trabajo. }\end{array}$ \\
& $\begin{array}{l}\text { Aspectos a mejorar en centro trabajo. } \\
\text { Tecnología del SAS. } \\
\text { Herramientas tecnológicas disponibles. }\end{array}$ \\
\hline Información y comunicación interna. & $\begin{array}{l}\text { Información suficiente. } \\
\text { Adecuación canales comunicación. } \\
\text { Fonales más utilizados. }\end{array}$ \\
\hline \multirow{2}{*}{ Cormación. } & Recibe formación necesaria. \\
\hline
\end{tabular}

\begin{tabular}{|c|l|}
\hline \multicolumn{2}{|c|}{ PERCEPCIÓN SATISFACCIÓNY CONSIDERACIÓN PROFESIONAL. } \\
\hline Motivación, participación y reconocimiento. & $\begin{array}{l}\text { Trabajo desarrollado es de su gusto (0-I0). } \\
\text { Sentimiento partícipe éxitos y fracasos SAS. } \\
\text { Sentimiento pertenencia equipo trabajo. } \\
\text { Sentimiento reconocimiento y valoración SAS. }\end{array}$ \\
\hline Clima laboral. & $\begin{array}{l}\text { Posibilidad expresar opiniones en grupo trabajo. } \\
\text { Vivencia de valores de equipo en el grupo de trabajo. } \\
\text { Reparto equilibrado carga trabajo. }\end{array}$ \\
\hline Satisfacción global & Grado satisfacción actividad laboral. \\
\hline
\end{tabular}

Fuente: elaboración propia con datos del estudio. 
Se recogieron 51 cuestionarios ( 20 de conserjes, 15 de auxiliares y 16 de educadorascuidadoras), lo que supone una representatividad de cada grupo respecto del total del $39 \%, 29 \%$ y $31 \%$ respectivamente y del conjunto de la población objeto de análisis, cercano al $81 \%$.

Después de cada sesión se desarrollaban grupos de discusión en los que los profesionales tenían oportunidad de expresar su valoración y opiniones, bien de los temas propuestos o de los que considerasen oportunos. Se celebraron nueve grupos de discusión, tres de cada categoría profesional analizada, integrados por 5-6 participantes y dos moderadores. Todos seguían la misma estructura formal y de contenidos, contando el equipo de moderadores con pautas comunes de actuación (Gutiérrez Brito, 2001, 2006).

Destacan dos características en nuestra muestra: la sobrecualificación y la feminización, hecho frecuente en el sector de los servicios sociales en España (Azpeitia Arman, 2003; Berasaluze Correa, 2009; Castelló Santamaría, 2011; Pazos Morán, 2011). La sobrecualificación se ha convertido en un fenómeno cada vez más habitual en el actual mercado de trabajo; suele relacionarse, entre otros factores, con el incremento de personas desempleadas en momentos de crisis económica y es más frecuente entre las mujeres que entre los hombres (García Montalvo \& Peiró Silla, 2009, 2011).

Se ha podido constatar una elevada satisfacción profesional de los colectivos en el desempeño de su trabajo, expresada con una valoración media de 7,86 puntos en una escala de o a 10 (desviación típica de 1,6). Se confirman estas positivas valoraciones cuando se les pregunta si les gusta su trabajo, alcanzando una puntuación media de 8,55 sobre 10 (d.t. 1,36). El Alfa de Cronbach que mide la consistencia interna para estos dos ítems es de 0,837 , indicando una muy buena fiabilidad (George \& Mallery, 2011). Las diferencias entre los grupos profesionales son mínimas y no significativas estadísticamente; tampoco se han encontrado diferencias ni correlaciones estadísticamente significativas en el análisis de la satisfacción global por sexo, edad, nivel de estudios o antigüedad profesional. Los tres grupos presentan un alto nivel de motivación laboral, con una valoración media de 8,5 (d.t.=1,36) sobre 10 , encontrándose todas las puntuaciones en el intervalo 6-10, con una moda global de 10.

Un análisis de los discursos evidencia también esa satisfacción profesional, resaltando la importancia de su labor en los servicios sociales y el carácter humano de su intervención. Son profesionales a los que les gusta lo que hacen, dan muestra de una elevada profesionalidad y una especial sensibilidad social en el desarrollo de su trabajo. Sus propuestas se orientan a mejorar la atención que realizan: una formación más específica y ajustada a sus puestos de trabajo, delimitación clara de funciones, aprovechamiento óptimo de sus capacidades, participación en la toma de decisiones sobre procesos que les afectan, encuentros periódicos para el intercambio de información y, en fin, una mayor consideración de su papel. También se perfilan como ámbitos a considerar las acciones para evitar el estrés y la sobrecarga de trabajo. Sus demandas denotan la aspiración de mejorar.

\section{Conclusiones}

El desarrollo de nuestros trabajos nos pemirte concluir que el análisis de satisfaccion de usuarios y profesionales ha abierto una línea de estudio de enorme interés y relevancia 
en el ámbito de los servicios sociales básicos. Investigaciones de este tipo se convierten en imprescindibles, tanto en los servicios sociales como en la Administración Pública en general, al dar voz a colectivos que suelen quedar al margen de la organización y de la toma de decisiones sobre los servicios.

Dede la perspectiva de la ciudadanía, se constata un importante desconocimiento sobre el papel de los servicios sociales, que se materializa en unas bajas expectativas sobre los mismos. Sin embargo, tras un paso por los mismos, destaca la elevada satisfacción con la atención recibida, especialmente con la figura del profesional. La adecuación a la demanda, desde un trato amable y sencillo se valora con unas elevadas puntuaciones cuando son consultados. También son claros los ámbitos de mejora, vinculados con los tiempos de respuesta, la burocracia vinculada a los procesos de gestión y los horarios de atención.

En cuanto a los colectivos profesionales analizados, se pone de manifiesto la importancia de reforzar la consideración del esencial papel de la primera línea de atención. Sus elevados niveles de implicación, junto con la alta satisfacción manifestada (con espacios de mejora de dificil detección si no son expresados por ellos mismos) nos sitúan ante un grupo altamente motivado y comprometido con la misión institucional.

Resaltar, en fin, la necesidad de potenciar el desarrollo de este tipo de estudios en el ámbito de los servicios sociales, generando instrumentos propios que respondan a las pecualiridades del sistema. La experiencia acumulada, aunque aún escasa y con limitada difusion, puede orientar el diseño de instrumentos que integren los elementos de análisis ya comentados, que se apuntan como esenciales. Así mismo sería importante profundizar en los aspectos que repetidamente se proponen como mejorables e indagar en otras variables sociodemográficas que puedan apuntar diferencias significativas en las valoraciones, dado que en los datos obtenidos no ha sido posible encontrar relaciones significativas con variables sociodemográficas.

Finalmente, se abre el reto de poder ofrecer series de datos, que permitan tanto un análisis comparado entre realidades distintas, como una comparación temporal, que muestre una imagen de la evolución de la satisfacción en el tiempo. Esta información, además de orientar los procesos de mejora, permitiría poner en valor la importancia de los servicios sociales para el bienestar de las comunidades y de la sociedad.

\section{Referencias Bibliográficas}

AENOR. (2003). Gestión de la calidad. Manual de normas UNE. Madrid: AENOR.

AEVAL. (2009). Guía para la evaluación de la calidad de los servicios públicos. Madrid: AEVAL.

AEVAL. (2014). Guía para la realización de estudios de análisis de la demanda y de evaluación de la satisfacción de los usuarios. Madrid: AEVAL.

Aguilar Hendrickson, M. (2013). Retos de los servicios sociales. Presupuesto y gasto público, 7, 271-290. 
Albrecht, K. (1998). Servicio al cliente interno: cómo solucionar la crisis de liderazgo en la gerencia intermedia. Barcelona: Paidós.

Alemán Bracho, C., \& Pérez Sánchez, M. (2000). Nuevas estrategias para el avance de los servicios sociales. Nueva gestión, análisis y evaluación. In J. A. Díaz Martínez \& M. J. Salvador Pedraza (Eds.), Nuevas perspectivas de los Servicios Sociales. Madrid: Universidad Nacional de Educación a Distancia.

Alemán Bracho, M. d. C., \& Trinidad Requena, A. (2012). Evaluación de servicios sociales. Cizur Menor, Navarra: Civitas.

Arenas Martínez, M. (2015). Calidad percibida en los servicios sociales de inclusión. Una aproximación desde el caso de Avilés (Asturias). Cuadernos de Trabajo Social, $28(1), 113-123$.

Ayuntamiento de Madrid. (2010). Cuestionario de satisfacción de usuarios de los centros de Servicios Sociales. Madrid: Dirección General de Servicios Sociales y Atención a la Dependencia.

Azpeitia Arman, M. C. (2003). Género e identidad profesional en los trabajadores sociales. Cuadernos de Trabajo Social(16), 147-170.

Barranco Expósito, C. (2000). Investigación sobre la calidad de vida y calidad del servicio en tres campos de Tenerife Cambio social, relaciones humanas, nuevas tecnologías: enfoques para una formación de futuro: [3 ${ }^{\circ}$ Congreso, Escuelas Universitarias de Trabajo Social] (Vol. 2, pp. 37-46). Zaragoza: Mira.

Barranco Expósito, C. (2004a). La intervención en Trabajo social desde la calidad integrada. Alternativas: cuadernos de trabajo social(12), 79-102.

Barranco Expósito, C. (2004b). Los profesionales de ayuda y el burnout. Trabajo Social y Salud(47), 27-35.

Barrera Algarín, E., Malagón Bernal, J. L., \& Sarasola Sánchez-Serrano, J. L. (2015). Trabajo social, su contexto profesional y el Síndrome de Burnout. Comunitania: Revista internacional de trabajo social y ciencias sociales(9), 51-71.

Bengoa, M. A., \& Celorio, M. J. (2003a). Los sistemas de acreditación como garantía de calidad. In M. L. Setién Santamaría \& E. Sacanell Berrueco (Eds.), La calidad en los servicios sociales: conceptos y experiencias (pp. 224-236). Valencia: Tirant lo Blanch.

Bengoa, M. A., \& Celorio, M. J. (2003b). Nuevas estrategias en la provisión de servicios humanos y clientes consumidores. Una visión desde el Trabajo Social. In M. L. Setién Santamaría \& E. Sacanell Berrueco (Eds.), La calidad en los servicios sociales: conceptos y experiencias (pp. 133-146). Valencia: Tirant lo Blanch.

Berasaluze Correa, A. (2009). El devenir del Trabajo Social en clave de género. Zerbitzuan: Revista de servicios sociales(46), 133-140. 
Bolton, R. N., \& Drew, J. H. (1991). A multistage model of customer's assessment of service quality and value. Journal of Consumer Research, 17, 375-384.

Cabrera Rodríguez, F. A., Donoso Vázquez, T., Aneas Álvarez, A., Campo Sorribas, J. d., \& Pi i Murugó, A. (2010). Valoración de la satisfacción de usuarios de programas sociales: propuesta de un modelo de análisis. Revista de Educación(351), 311336.

Cardozo, R. N. (1965). An empirical study of custumer effort, expectation, and satisfaction. Journal of Marketing research, 2, 244-249.

Casado Pérez, D. (2007). Objetivos permanentes y enfoques de los servicios sociales Perfeccionamiento de los servicios sociales en España: informe con ocasión de la Ley sobre autonomía y dependencia (pp. 255-288).

Castelló Santamaría, L. (2011). Trabajo de cuidado, género y clase social. Remedios desiguales a un problema común. Sociología del trabajo(73), 24-41.

Castillo Alonso, J. J., \& Prieto Rodríguez, C. (1981). Una técnica subjetiva de investigación en condiciones de trabajo: las encuestas de Satisfacción. Reis, 13(13), 49-63.

Castillo Alonso, J. J., \& Prieto Rodríguez, C. (1983). Condiciones de trabajo: hacia un enfoque renovador de la sociología del trabajo. Madrid: Centro de Investigaciones Sociológicas.

Cosano Rivas, F., Hombrados Mendieta, M. I., \& Castilla Mora, R. (2014). Un modelo explicativo de la influencia del apoyo social en el burnout y la satisfacción laboral en el Trabajo Social. El trabajo social ante el reto de la crisis y la educación superior [Recurso electrónico], 927-934.

Chiang Vega, M. M., Salazar Botello, C. M., \& Núñez Partido, A. (2007). Climay satisfacción laboral en instituciones públicas: adaptación y ampliación de un instrumento. In J. C. Ayala Calvo (Ed.), Conocimiento, innovación y emprendedores : camino al futuro. Logroño: Universidad de La Rioja.

De La Red Vega, N., \& Conde Rodríguez, C. (2000). Los proyectos de intervención integral en el ámbito de los Servicios Sociales en cooperación con otras redes y sistemas de bienestar social. In J. A. Y. S. P. Diaz Martinez, M.J. (Ed.), Nuevas perspectivas de los Servicios Sociales. Madrid: Universidad Nacional de Educación a Distancia.

Del Pozo Herráiz, B., \& Sánchez Pérez, M. C. (2010). Trabajando por la calidad desde los servicios sociales básicos: una experiencia. Miradas a lo social [Recurso electrónico]: procesos y problemas sobre los que actúa el Trabajo Social, 109120.

Domínguez González, J., \& Jaureguibehere, V. (2012). Trabajo social y Síndrome de Burnout: reflexiones sobre intervención profesional actual. Documentos de trabajo social: Revista de trabajo y acción social(51), 140-144.

Donabedian, A. (1989). La calidad de la asistencia. ¿Cómo podría ser evaluada? Revista JANO, 864, 103-110. 
Esteban Ramiro, B. (2014). Una aproximación a la influencia de la Inteligencia Emocional Percibida en su relación con los niveles de Burnout y Engagement en el desempeño del Trabajo Social. Azarbe: Revista Internacional de Trabajo Social y Bienestar(3), 123-131.

Facal Fondo, T. (2012). Prevalencia del Síndrome de Burnout en trabajadores sociales de los servicios sociales comunitarios. Portularia: Revista de Trabajo Social(12), 59-69.

Fantova Azcoaga, F. (2008). Sistemas públicos de servicios sociales: nuevos derechos, nuevas respuestas. Bilbao: Universidad de Deusto.

Fernández Guerrero, R., \& Martínez Moreno, A. (2003). Personas, conocimiento e innovación en servicios sociales. In M. L. Setién Santamaría \& E. Sacanell Berrueco (Eds.), La calidad en los servicios sociales: conceptos y experiencias (pp. 59-93). Valencia: Tirant lo Blanch.

Fuente Roldán, I. N. d. l., \& Sánchez Moreno, E. (2012). Trabajo social, síndrome de estar quemado por el trabajo y malestar psíquico: un estudio empírico en una muestra de trabajadores sociales de la Comunidad de Madrid. Portularia: Revista de Trabajo Social(12), 121-130.

Fuentes García, F. J., Sánchez Cañizares, S. M., \& Artacho Ruiz, C. (2008). Rankings internacionales de satisfacción laboral. Capital humano: revista para la integración y desarrollo de los recursos humanos, 21(219), 60-70.

Fundación FOESSA (2009). VI Informe sobre exclusión y desarrollo social en España :2008: Fundación Fomento de Estudios Sociales y de Sociología Aplicada, FOESSA.

García Montalvo, J., \& Peiró Silla, J. M. (2009). Análisis de la sobrecualificación y la flexibilidad laboral: observatorio de inserción laboral de los jóvenes 2008. Valencia: Instituto Valenciano de Investigaciones Económicas (IVIE).

García Montalvo, J., \& Peiró Silla, J. M. (2011). Crisis económica e inserción laboral de los jóvenes: Resultados del Observatorio de Inserción Laboral de los Jóvenes 2011. Valencia: Instituto Valenciano de Investigaciones Económicas (IVIE).

García Pineda, M. L., \& Gago Cabrera, M. S. (2010). Estudio del perfil de usuarios de un centro de servicios sociales Una Europa social y plural [Recurso electrónico]: actas del VII Congreso Estatal de Escuelas Universitarias de Trabajo Social (pp. 148). Granada: Universidad de Granada.

García Sánchez, F. A., Mirete Ruiz, A. B., Marín Oller, C., \& Romero, L. (2008). Satisfacción del cliente familia en atención temprana: valoración de la importancia que otorgan a distintos aspectos del servicio. Siglo Cero: Revista Española sobre Discapacidad Intelectual, 39(227), 55-74.

George, D., \& Mallery, P. (2011). SPSS for Windows step by step : a simple guide and reference 18.o update (11th ed.). Boston, MA: Allyn \& Bacon/Pearson. 
Giménez Bertomeu, V. M., \& Doménech López, Y. (2012). Expertos por la experiencia y expertos por la profesión. Visiones sobre la práctica profesional en los Servicios Sociales de Atención Primaria. Cuadernos de Trabajo Social, 25(2), 439-449.

Giménez Bertomeu, V. M., Lillo Beneyto, A., Doménech López, Y., \& Lorenzo García, J. (2012). La Calidad en los servicios sociales de atención primaria desde la perspectiva de las personas usuarias. Portularia: Revista de Trabajo Social(12), 61-71.

Gómez García, R. (2013). Influencia de factores personales y organizacionales en la satisfacción laboral de los trabajadores sociales. Portularia: Revista de Trabajo Social(13), 25-37.

Gutiérrez Brito, J. (2001). Elementos no-técnicos para la conducción de un grupo de discusión. Empiria: Revista de metodología de ciencias sociales(4), 121-144.

Gutiérrez Brito, J. (2006). El grupo de discusión La investigación social del turismo: perspectivas y aplicaciones (pp. 163-175). Madrid: Thomson.

Hackman, J. R., \& Oldham, G. R. (1976). Motivation through the design of work: test of a theory. Organizational Behavior and Human Performance, 16, 250-279. .

Herzberg, F., Mausner, B., \& Snyderman, B. (2010). The motivation to work (12th ed. ed.). New Brunswick: : Transaction Publishers.

Imserso. (1998). Atención a las personas mayores que viven en residencias. Protocolos de intervención psicosocial y sanitaria. Madrid: Ministerio de Trabajo y Asuntos Sociales.

IMSERSO. (1999). Analizando la calidad de servicio. Calidad de vida, calidad de servicio. Madrid: IMSERSO.

Juanes, B., \& Blanco, J. (2001). El Gato de Alicia. Modelos de calidad en la administración pública. Madrid: Díaz de Santos.

Literas Rondón, L., Navarro, A., \& Fontanals de Nadal, D. (2010). Diseño y validación de una escala de satisfacción y calidad de vida para usuarios de centros residenciales y sociosanitarios. Revista española de geriatría y gerontología: Organo oficial de la Sociedad Española de Geriatría y Gerontología, 45(6), 320-325.

Lucas Marín, A. (1981). Una aproximación sociológica al estudio de la satisfacción en el trabajo en la provincia de Santander. Reis, 13(13), 65-100.

MAP. (2006). Guía de orientación para la realización de estudios de análisis de la demanda y de encuestas de satisfacción. Madrid: Ministerio de Administraciones Públicas.

Martín López, M. d. C., Padial Díaz, M. d. C., Romero Gallardo, R., \& Serrano Guerrero, R. (1996). Satisfacción laboral de los trabajadores sociales de Málaga (I). Documentos de trabajo social: Revista de trabajo y acción social(8), 15-27. 
Martínez Tur, V., Ramos López, J., Marzo, J. C., \& Peiró Silla, J. M. (2002). La satisfacción del usuario desde el modelo de la confirmación de expectativas: respuesta a algunos interrogantes. Psicothema, 14(4), 765-770.

Medina Tonero, M. E. (2000). Evaluación de la calidad asistencial del servicio de ayuda a domicilio en el Ayuntamiento de Murcia. Murcia: Universidad de Murcia.

Medina Tonero, M. E. (2003). La calidad de vida en la gestión de la calidad: el servicio de ayuda a domicilio. In M. L. Setién Santamaría \& E. Sacanell Berrueco (Eds.), La calidad en los servicios sociales: conceptos y experiencias (pp. 189-196). Valencia: Tirant lo Blanch.

Medina Tornero, M. E. (1997). Evaluation of the quality of assistance in social services. Psychology in Spain(1), 3-9.

Medina Tornero, M. E., \& Medina Ruiz, E. (2011). Análisis de la calidad percibida en usuarios/as de servicios sociales comunitarios. Zerbitzuan: Gizarte zerbitzuetarako aldizkaria $=$ Revista de servicios sociales(50), 85-100.

Moya Vaquero, E. (2000). Herramientas para gestionar la calidad en un centro de Servicios Sociales. Revista de Servicios Sociales Y Política Social(49), 85-90.

Parasuraman, A., Zeithmal, V., \& Berry, L. (1994). Reassessment of expectations as a comparison standard in measuring service quality: implications for further research. Journal of Marketing research, 58, 111-124.

Pazos Morán, M. (2011). Roles de género y políticas públicas. Sociología del trabajo(73), 5-23.

Peña Salas, E. d. l. (2014). Análisis de la satisfacción y de la calidad percibida por las personas atendidas en los Servicios Sociales Comunitarios del Centro Municipal de Servicios Sociales Delicias del Ayuntamiento de Zaragoza. Cuadernos de Trabajo Social, 27(1), 115-125.

Pérez Cosín, J. V., \& Deslauriers, J.-P. (2004). El reto del conocimiento en la práctica del Trabajo Social. Cuadernos de Trabajo Social(17), 195-210.

Pérez Menayo, V. (2005). La calidad social, un nuevo paradigma en la Unión Europea Madrid: Ministerio de Trabajo y Asuntos Sociales.

Peters, T. J., \& Waterman, R. H. (1991). En busca de la excelencia : lecciones de las empresas mejor gestionadas de Estados Unidos. Barcelona: Folio.

Pillinger, J., \& Fundación Europea para la Mejora de las Condiciones de Vida y de Trabajo. (2001). Quality in social public services. Luxembourg: Office for Official Publications of the European Communities.

Prats Darder, P. (2005). Métodos para medir la satisfacción del cliente. Madrid: AENOR. 
Robles Ríos, P., \& Lara Martínez, F. (2009). Evaluación del Servicio de Ayuda a Domicilio en Alcorcón: diagnóstico para la mejora de la calidad del servicio. Cuadernos de Trabajo Social(22), 259-276.

Salgado Velo, J., Iglesias, M., \& Remeseiro Rioboo, C. (1996). Clima organizacional y satisfacción laboral en una pyme. Psicothema, 8(2), 329-335.

Salvador Pedraza, M. J. (2000a). Calidad y servicios sociales. In J. A. Diaz Martinez \& M. J. Salvador Pedraza (Eds.), Nuevas perspectivas de los Servicios Sociales. Madrid: Universidad Nacional de Educación a Distancia.

Salvador Pedraza, M. J. (200ob). Los retos de la calidad en los Servicios Sociales. Revista de Servicios Sociales Y Política Social(50), 27-54.

Salvador Pedraza, M. J. (2003). Criterios de calidad en los servicios de atención a las personas. In M. L. Setién Santamaría \& E. Sacanell Berrueco (Eds.), La calidad en los servicios sociales: conceptos y experiencias (pp. 147-175). Valencia: Tirant lo Blanch.

Sánchez Aguado, A. (2011). La calidad en la gestión de los Servicios Sociales Municipales: algunas experiencias de INTRESS. TS nova: trabajo social y servicios sociales(3), 49-54.

Sánchez Cañizares, S. M. (2006). Diseño de un modelo causal de satisfacción laboral: aplicación al sector servicios. Córdoba: Universidad de Córdoba.

Sánchez Pérez, M. C., \& Aguilar, J. M. (2016). Satisfacción profesional en los servicios sociales municipales: los trabajadores y trabajadoras de primera línea. Sociología del trabajo(86), 64-86.

Sánchez, R., Martínez Tur, V., González Morales, M. G., Ramos López, J., \& Peiró Silla, J. M. (2009). Un análisis transnivel de las relaciones de la calidad de servicio y la confirmación de expectativas con la satisfacción de los usuarios. Psicothema, 21(3), 421-426.

Siis. Dokumentazio eta Ikerketa Zentroa Centro de Documentación y Estudios. (2010). La situación de los Servicios Sociales de Base en la Comunidad Autónoma del País Vasco. Zerbitzuan: Gizarte zerbitzuetarako aldizkaria $=$ Revista de servicios sociales(47), 7-36.

Tornero, M. E. M., Ruiz, E. M., \& Miralles, R. V. (2011). Calidad percibida en usuarios/ as de servicios sociales comunitarios Paper presented at the III Congreso Anual de la REPS. Los actores de las políticas sociales en contextos de transformación., Pamplona.

Trinidad Requena, A. (2010). La evaluación participativa en la Nueva Gestión Pública. RIO: Revista Internacional de Organizaciones(5), 75-107.

Trinidad Requena, A., \& García Moreno, J. M. (2012). Las lógicas de la evaluación de programas de invervención iimposición o participación? Azarbe: Revista Internacional de Trabajo Social y Bienestar(1), 111-126. 
Vavra, T. G. (2002). Cómo medir la satisfacción del cliente según la ISO 9oo1:2000: Madrid : Fundación Confemetal, 2002.

Villegas Periñán, M. M., \& Rosa Díaz, I. M. (2003). La calidad asistencial: concepto y medida. Dirección y organización: Revista de dirección, organización y administración de empresas,(29), 50-58.

Viñas, V. (2005). Satisfacción del cliente, calidad y evaluación: un análisis crítico. en Revista del CLAD Reforma y Democracia(32).

Vuori, H. V., Saturno Hernández, P., Delgado Vila, R., \& Suñol Sala, R. (1996). El Control de calidad en los servicios sanitarios : conceptos y metodología. Barcelona, etc.: Masson.

Zamudio Igami, M. P. (2005). El uso del SERVQUAL en la verificación de la calidad de los servicios de unidades de información: el caso de la Biblioteca del IPEN. Revista Interamericana de Bibliotecología, 28(2), 177-191.

Zeithaml, V.A., \& Parasuraman, A. (1994). Reassessment of expectations as a comparison standard in measuring service quality: Implications. Journal of marketing: A quarterly publication of the american marketing association, 58(1), 111-124.

Zeithaml, V. A., Parasuraman, A., \& Berry, L. L. (1990). Delivering quality service : balancing customer perceptions and expectations. New York: The Free Press.

Zeithaml, V. A., Parasuraman, A., \& Berry, L. L. (1993). Calidad total en la gestión de servicios : cómo lograr el equilibrio entre las percepciones y las expectativas de los consumidores. Madrid: Díaz de Santos. 